\title{
Development of a commonized methodology for measuring brake wear particles - current status within the PMP IWG
}

Theodoros Grigoratos, G. Martini, Joint Research Centre (JRC), European Commission (EC), Italy

This manuscript is not available according to publishing restriction.

Thank you for your understanding. 